\title{
Clinical Effects of Commiphora Myrrha in Oral and Dental Medicine, A Mini Review
}

\author{
Adnan A Almaghlouth* \\ Periodontics Section, Dentistry Adminstration, King Fahad Medical City, Riyadh, Kingdom of Saudi Arabia
}

*Corresponding author: Adnan A Almaghlouth, Periodontics Section, Dentistry Adminstration, King Fahad Medical City. P.0. Box.59046, Riyadh 11525, Kingdom of Saudi Arabia.
Received Date: February 10, 2021

Published Date: February 16, 2021

\section{Mini Review}

The United States Department of Agriculture USDA recognizes Commiphora myrrha, named myrrh, African myrrh, herbal myrrh, Somali myrrh, or gum myrrh as a tree in the Burseraceous family [1]. It is one of the primary trees used in the production of myrrh, a resin made from dried tree sap. According to the world agroforestry center, the tree exists in the middle east (Oman, Yemen) and in some African countries (Djibouti, Ethiopia, Somalia, Northeast Kenya). In Arabic, it is named 'mur', which means bitter. It is the gum of the myrrh tree. Its oil is called oleoresin. It famously comes from Mecca, so it is called 'Mur Makki' [2]. According to Tonkal, Morsy [3], the genus Commiphora is composed of more than 200 species, and intimidated as a natural drug to treat pain, skin infections, inflammatory conditions, diarrhea, and periodontal diseases [3]. Traditional practice and evidence-based research have supported that these characteristics are directly attributable to terpenoids (especially furanosesquiterpenes), the active compounds present in myrrh essential oil [4].

The literature showed Only few studies evaluated the beneficial effects of the C. Myrrhaare when used as a drug for oral and dental diseases. Al-Madi and coworkers in 2020 compared the antimicrobial efficacy of the extract of Commiphora myrrha, against Enterococcus faecalis and Fusobacterium nucleatum [5]. 140 extracted human premolar teeth were instrumented and immersed in bacterial suspension of E. faecalis or F. nucleatum. Prepared teeth were then randomly assigned to the following four subgroups: myrrh ethanol extract $(30 \mathrm{mg} / 300 \mathrm{uL}), 2.5 \% \mathrm{NaOCl}$ DMSO as negative control, and $1 \mathrm{~g}$ of Cefotaxime antibiotic injection as positive control and incubated for 30 and 60 minutes. The author found no significant difference in the antimicrobial effect of $30 \mathrm{mg} / 300 \mu \mathrm{L}$ myrrh from that of sodium hypochlorite $(\mathrm{NaOCl})$ against both bacterial species confirming its effective antimicrbial effect in root canal infection. In another interesting in-vitro study evaluated the effect of myrrh on the strength of three strong suture materials used in oral surgeries: silk, polyglactin 910, and polytetrafluoroethylene. The author found all three suture materials lost tensile strength when exposed to $100 \%$ myrrh oil [6]. Indeed, this imply the potent effect of this oil at all surfaces used.

Saeedi and coworkers conducted a clinical trial in 2003 to evaluate the outcomes following myrrh use on the oral mucosa. Test groups received toothpastes containing either myrrh alone, or myrrh with chamomile for daily use, while control groups received placebo toothpaste. The researcher found a significant improvement in gingival bleeding in subjects who used myrrhcontaining toothpaste compared to controls suggesting that myrrhcontaining toothpastes may be helpful as part of the treatment of patients with gingival lesions [7]. In a recent randomized controlled clinical trial, C. Myrrha was tasted in the form of a mouth rinse to evaluate its clinical effects following tooth extractions [8]. 40 subjects were randomized to two equal groups. The test group received $1.25 \mathrm{gm}$ of grinded myrrh to be dissolved by the patient in a cup of $250 \mathrm{ml}$ of warm water and prescribed for rinsing twice daily for 7 days. Control group rinsed with saline mouthwash $(0.90 \%$ $\mathrm{w} / \mathrm{v}$ of $\mathrm{NaCl}$ ). Clinical evaluation included systemic findings like fever and fatigue as well as local findings including color, swelling, tenderness, odor, discharge, bleeding, limited mouth opening, and socket size changes. The study found the majority of the patients 
did not have significant complaints, symptoms, infection, or severe abnormalities during the postoperative assessment in both groups confirming the safety use of Myrrha as a post-operative antimicrobial mouth rinse.

The present review addressed important findings about C. Myrrha. There is a growing evidence that it may exert adequate anti-inflammatory as well as antimicrobial effects in oral and dental tissues. Still, the evidence so far is limited, and further studies are recommended to address clearly its antimicrobial effects on different periodontal pathogens including Aggregatibacter actinomycetemcomitans and Porphyromonas gingivalis. Additionally, the research has to focus on the adjusted duration for its optimal use and weather to be provided adjunctive to additional therapy or as a solo drug. Moreover, Gingival and periodontal parameters have to be studied clearly when C. Myrrha is tested at clinical trials. This will help streamlining the positive clinical outcomes and provide excellent comparison with other oral antimicrobial and anti-inflammatory oral mouth rinses. As like other drugs used for the same clinical purposes, adverse events have to be clearly documented at both, short- and long-term basis. The concentration is another important data that needs more investigation in the clinical research as it showed impact on the strength of surgical sutures and flap closure [6].

\section{Acknowledgement}

The author would like to thank the Research Center at King Fahd Medical City and the Saudi Society of Periodontology for their valuable support in the preparation of this manuscript.

\section{Conflict of Interest}

No conflict of interest.

\section{References}

1. a b c d e Commiphora myrrha (2019), Germplasm Resources Information Network (GRIN). Agricultural Research Service (ARS), United States Department of Agriculture (USDA).

2. Species Information (2009).

3. Abdulkader M D Tonkal, Tosson A Morsy (2008) An update review on Commiphora molmol and related species. J Egypt Soc Parasitol 38(3): 763-796.

4. Nomicos Effie Y H (2007) Myrrh: medical marvel or myth of the Magi? Review. Holist Nurs Pract 21(6): 308-323.

5. Al-Madi EM, Almohaimede AA, Al-Obaida MI, Awaad AS (2009) Comparison of the Antibacterial Efficacy of Commiphora molmol and Sodium Hypochlorite as Root Canal Irrigants against Enterococcus faecalis and Fusobacterium nucleatum. Evid Based Complement Alternat Med 6916795

6. Mohammed A Alshehri, Jagan Kumar Baskaradoss, Amrita Geevarghese, Ravikumar Ramakrishnaiah, Dimitris N Tatakis (2015) Effects of myrrh on the strength of suture materials: an in vitro study. Dent Mater J 34(2): 148-153.

7. Saeedi M, Azadbakht M, Semnani K, Khandan M (2003) Formulation of herbal toothpaste from chamomile and myrrh, a preliminary clinical evaluation of bleeding gum. J Mazandaran Univ Med Sci 13: 61-69.

8. Eid RAA (2021) Efficacy of Commiphora myrrh mouthwash on early wound healing after tooth extraction: A randomized controlled trial. Saudi Dent J 33(1): 44-54. 